\title{
SIMULATION OF CNT COMPOSITES USING FAST MULTIPOLE BEM
}

\author{
Zhen-Han Yao \\ Department of Engineering Mechanics, Tsinghua University, Beijing, P.R.C., demyzh@tsinghua.edu.cn \\ Jun-Dong Xu \\ Department of Engineering Mechanics, Tsinghua University, Beijing, P.R.C.
}

\section{Hai-Tao Wang}

Institute of Nuclear and New Energy Technology, Tsinghua University, Beijing, P.R.C.

Xiao-Ping Zheng

Department of Engineering Mechanics, Tsinghua University, Beijing, P.R.C.

Follow this and additional works at: https://jmstt.ntou.edu.tw/journal

Part of the Electrical and Computer Engineering Commons

\section{Recommended Citation}

Yao, Zhen-Han; Xu, Jun-Dong; Wang, Hai-Tao; and Zheng, Xiao-Ping (2009) "SIMULATION OF CNT COMPOSITES USING FAST MULTIPOLE BEM," Journal of Marine Science and Technology. Vol. 17: Iss. 3, Article 5.

DOI: $10.51400 / 2709-6998.1956$

Available at: https://jmstt.ntou.edu.tw/journal/vol17/iss3/5

This Research Article is brought to you for free and open access by Journal of Marine Science and Technology. It has been accepted for inclusion in Journal of Marine Science and Technology by an authorized editor of Journal of Marine Science and Technology. 


\section{SIMULATION OF CNT COMPOSITES USING FAST MULTIPOLE BEM}

Acknowledgements

Financial supports for the project from the National Natural Science Foundation of China, under grant No. 10472051, and for a project from Schlumberger Technologies (Beijing) Ltd., are gratefully acknowledged. 


\title{
SIMULATION OF CNT COMPOSITES USING FAST MULTIPOLE BEM
}

\author{
Zhen-Han Yao*, Jun-Dong Xu*, Hai-Tao Wang**, and Xiao-Ping Zheng*
}

Key words: fast multipole BEM, CNT composites, elastic property, thermal property, electric property.

\begin{abstract}
This paper addresses some applications of the fast multipole boundary element method on the simulation of CNT composites, including the simulation of elastic, thermal and electric properties. The carbon nanotubes are treated as effective elastic fibers for the simulation of elastic property, and treated as straight fibers with high thermal conductivity and high electric conductivity for the simulation of thermal and electric properties respectively. The numerical examples are compared with other numerical results and experimental data published in literature. To reduce the difference between numerical and experimental value of effective elastic modulus, two approaches with nonideal interfacial condition or additional interfacial layer have been introduced. Numerical tests clearly demonstrate the potential of the FM-BEM for large scale simulation of CNT composites.
\end{abstract}

\section{INTRODUCTION}

In recent years, the fast multipole boundary element method (FM-BEM) has attracted the interest of many researchers. It is proved that FM-BEM can solve some very large scale problems, which are difficult to be solved by FEM and other methods. One of the main tasks in this field is to find such practical applications in engineering and sciences. At the same time, CNT composites have attracted considerable interest in various industrial applications because of the unusual physical properties of carbon nanotubes, e.g. unusually high elastic modulus, thermal and electric conductivity. The simulation of $\mathrm{CNT}$ composites is perhaps one of the valuable practical applications of FM-BEM.

Based on the previous investigation of the large scale simulation of particle- and fiber-reinforced composite materials using FM-BEM $[3,7,8]$, the carbon nanotubes are treated as effective elastic fibers for the simulation of elastic property,

Author for correspondence: Zhen-Han Yao (e-mail: demyzh@tsinghua.edu.cn). *Department of Engineering Mechanics, Tsinghua University, Beijing, P.R.C. **Institute of Nuclear and New Energy Technology, Tsinghua University, Beijing, P.R.C. and treated as straight fibers with high thermal conductivity and high electric conductivity for the simulation of thermal and electric properties respectively. To enhance the efficiency, the repeated identical subdomain approach is applied, and for large scale simulation the parallel FM-BEM computation is performed.

Some typical numerical examples are given to verify the applicability of the presented approach. The numerical results of effective properties obtained are compared with the corresponding numerical and experimental data published in literature $[1,2,4-6]$. To reduce the difference between numerical and experimental value of effective elastic modulus, two approaches with nonideal interfacial condition or additional interfacial layer have been introduced, and their effectiveness has been verified as well.

\section{SIMULATION OF ELASTIC PROPERTY}

For the simulation of elastic property, an effective continuum model of CNT is adopted, the CNT composite is modeled as elastic fiber reinforced composite with ideal interfacial condition. It is simulated using large-scale parallel computation of fast multipole boundary element method, wherein the repeated identical subdomain approach is applied. Numerical examples are given to show the effectiveness of the presented approach, and the comparison of the numerical and experimental results has shown remarkable difference. To reduce such difference two revised models with nonideal interfacial conditions are presented: one with zero-thickness elastic interface, and another with embedded elastic interfacial layer. The numerical results have shown the effect of the presented revised models.

\section{Effective Continuum Model of CNT Composite with Ideal Interfacial Condition}

In the effective continuum model of CNT composite, each CNT is regarded as an effective elastic fiber of circular cylinder with hemispherical ends as shown in Fig. 1(a). As suggested by Odegard [5], the real volume of CNT is taken as $37 \%$ of effective elastic fiber's volume. The length and diameter of the fiber are denoted as $L$ and $D$. In the BE computation, each fiber is divided into BE mesh as shown in Fig. 1(b), wherein triangular constant elements are applied. 


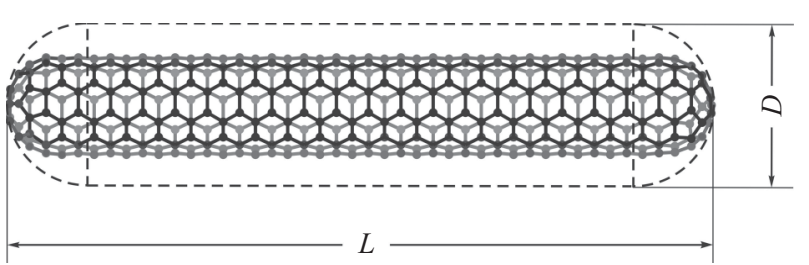

(a) effective elastic fiber of CNT

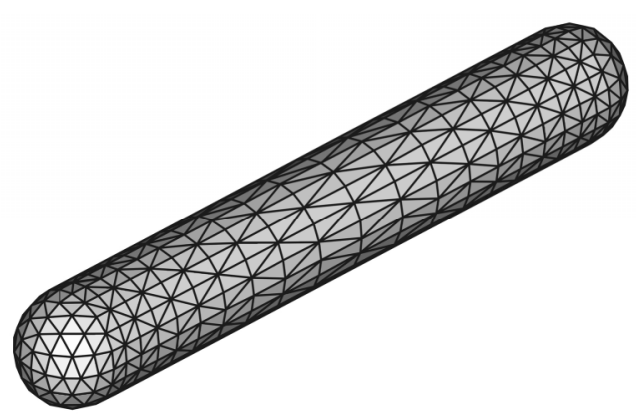

(b) BE mesh of an CNT fiber

Fig. 1. An effective elastic fiber of CNT and its BE mesh.

For the simulation of CNT composite, the representative volume element (RVE) with randomly distributed CNT fibers is applied, which is similar to that used for the simulation of conventional fiber-reinforced composites [3, 7]. A RVE containing 200 randomly distributed and oriented CNTs is shown in Fig. 2, wherein each CNT is assumed to be identical, with the same geometry and the same BE mesh, and the interfacial conditions between matrix and CNTs is assumed to be ideal.

\section{Repeated Identical Subdomain Approach of BEM}

For the RVE shown in Fig. 2, the boundary integral equations of the matrix and the $k$-th CNT fiber can be expressed respectively as

$$
\begin{gathered}
C_{i j}(x) u_{j}(x)+\int_{S_{0}+\sum S_{k}} T_{i j}^{0}(x, y) u_{j}(y) d S(y) \\
=\int_{S_{0}+\sum S_{k}} U_{i j}^{0}(x, y) t_{j}(y) d S(y) \\
C_{i j}(x) u_{j}(x)+\int_{S_{k}} T_{i j}^{k}(x, y) u_{j}(y) d S(y) \\
=\int_{S_{k}} U_{i j}^{k}(x, y) t_{j}(y) d S(y)
\end{gathered}
$$

where $V_{0}$ is the subdomain of the matrix, $V_{1}, V_{2}, \ldots, V_{k}, \ldots, V_{n}$ are the subdomains of CNTs, $S_{0}$ is the outer boundary of the matrix, and $S_{k}$ is the CNT-matrix interface of the $k$-th CNT. In addition, $S_{0}^{U}$ and $S_{0}^{T}$ are outer boundary parts of the matrix with given displacement and given traction, respectively. CNTs are randomly placed in the cuboid matrix, without connection between each two CNTs and without intersection with the surface of the matrix.

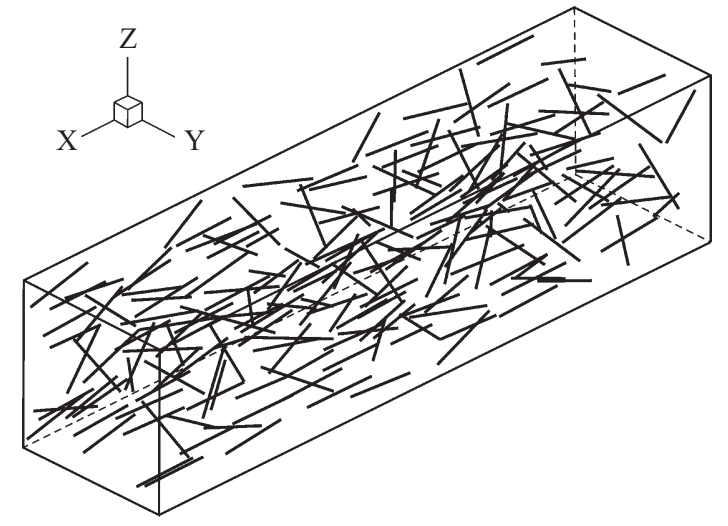

Fig. 2. A RVE containing 200 randomly oriented CNTs.

After discretization, Eqs. (1) and (2) can be written into matrix forms as follows,

$\left.\begin{array}{c}{\left[\begin{array}{ccccccc}\boldsymbol{A}_{11}^{0} & \boldsymbol{A}_{12}^{0} & \boldsymbol{A}_{13}^{1} & \cdots & \boldsymbol{A}_{13}^{k} & \cdots & \boldsymbol{A}_{13}^{n} \\ \boldsymbol{A}_{21}^{0} & \boldsymbol{A}_{22}^{0} & \boldsymbol{A}_{23}^{1} & \cdots & \boldsymbol{A}_{23}^{k} & \cdots & \boldsymbol{A}_{23}^{n} \\ \boldsymbol{A}_{31}^{1} & \boldsymbol{A}_{32}^{1} & \boldsymbol{A}_{33}^{11} & \cdots & \boldsymbol{A}_{33}^{1 k} & \cdots & \boldsymbol{A}_{33}^{1 n} \\ \vdots & \vdots & \vdots & \vdots & \vdots & \vdots & \vdots \\ \boldsymbol{A}_{31}^{k} & \boldsymbol{A}_{32}^{k} & \boldsymbol{A}_{33}^{k 1} & \cdots & \boldsymbol{A}_{33}^{k k} & \cdots & \boldsymbol{A}_{33}^{k n} \\ \vdots & \vdots & \vdots & \vdots & \vdots & \vdots & \vdots \\ \boldsymbol{A}_{31}^{n} & \boldsymbol{A}_{32}^{n} & \boldsymbol{A}_{33}^{n 1} & \cdots & \boldsymbol{A}_{33}^{n k} & \cdots & \boldsymbol{A}_{33}^{n n}\end{array}\right]\left\{\begin{array}{c}\boldsymbol{U}_{0} \\ \boldsymbol{T}_{0} \\ \boldsymbol{U}_{1}^{0} \\ \vdots \\ \boldsymbol{U}_{k}^{0} \\ \vdots \\ \boldsymbol{U}_{n}^{0}\end{array}\right\}} \\ -\left[\begin{array}{cccccc}\boldsymbol{B}_{13}^{1} & \cdots & \boldsymbol{B}_{13}^{k} & \cdots & \boldsymbol{B}_{13}^{n} \\ \boldsymbol{B}_{23}^{1} & \cdots & \boldsymbol{B}_{23}^{k} & \cdots & \boldsymbol{B}_{23}^{n} \\ \boldsymbol{B}_{33}^{11} & \cdots & \boldsymbol{B}_{33}^{1 k} & \cdots & \boldsymbol{B}_{33}^{1 n} \\ \vdots & \vdots & \vdots & \vdots & \vdots \\ \boldsymbol{B}_{33}^{k 1} & \cdots & \boldsymbol{B}_{33}^{k k} & \cdots & \boldsymbol{B}_{33}^{k n} \\ \vdots & \vdots & \vdots & \vdots & \vdots \\ \boldsymbol{B}_{33}^{n 1} & \cdots & \boldsymbol{B}_{33}^{n k} & \cdots & \boldsymbol{B}_{33}^{n n}\end{array}\right]\left\{\begin{array}{c}\boldsymbol{T}_{1}^{0} \\ \vdots \\ \boldsymbol{T}_{k}^{0} \\ \vdots \\ \boldsymbol{T}_{n}^{0}\end{array}\right\}=\left[\begin{array}{cc}\boldsymbol{B}_{11}^{0} & \boldsymbol{B}_{12}^{0} \\ \boldsymbol{B}_{21}^{0} & \boldsymbol{B}_{22}^{0} \\ \boldsymbol{B}_{31}^{1} & \boldsymbol{B}_{32}^{1} \\ \vdots & \vdots \\ \boldsymbol{B}_{31}^{k} & \boldsymbol{B}_{32}^{k} \\ \vdots & \vdots \\ \boldsymbol{B}_{31}^{n} & \boldsymbol{B}_{32}^{n}\end{array}\right]\left\{\overline{\boldsymbol{T}}_{0}\right. \\ \overline{\boldsymbol{U}}_{0}\end{array}\right\}$

$$
\boldsymbol{H}^{\mathrm{f}} \boldsymbol{U}_{k}^{\mathrm{f}}=\boldsymbol{G}^{\mathrm{f}} \boldsymbol{T}_{k}^{\mathrm{f}}
$$

where $\boldsymbol{U}_{0}$ and $\overline{\boldsymbol{T}}_{0}$ stand for the unknown nodal displacements and given nodal tractions on $S_{0}^{T}$, respectively, $\boldsymbol{T}_{0}$ and $\overline{\boldsymbol{U}}_{0}$ the unknown nodal tractions and given nodal displacements on $S_{0}^{U}$, respectively, $\boldsymbol{U}_{k}^{0}$ and $\boldsymbol{U}_{k}^{\mathrm{f}}$ stand for the unknown nodal displacements on $S_{k}$ for the matrix and the $k$-th CNT fiber, respectively. $\boldsymbol{T}_{k}^{0}$ and $\boldsymbol{T}_{k}^{\mathrm{f}}$ the unknown nodal tractions on $S_{k}$ for the matrix and the $k$-th CNT fiber, respectively. Submatrices of $\boldsymbol{A}$ and $\boldsymbol{B}$ in (3) consist of entries for the matrix material subdomain, and $\boldsymbol{H}^{\mathrm{f}}, \boldsymbol{G}^{\mathrm{f}}$ in (4) for the CNT fiber subdomain.

Introducing the CNT-matrix interface conditions, the interface tractions can be computed by interface displacements 
using incidence matrix $\boldsymbol{D}^{\mathrm{f}}$ and transform matrix $\boldsymbol{M}_{k}$ between the local and global coordinates of the $k$-th interface boundary,

$$
\boldsymbol{T}_{k}^{0}=-\boldsymbol{M}_{k} \boldsymbol{D}^{\mathrm{f}}\left(\boldsymbol{M}_{k}\right)^{-1} \boldsymbol{U}_{k}^{0} \quad \boldsymbol{D}^{\mathrm{f}}=\left(\boldsymbol{G}^{\mathrm{f}}\right)^{-1} \boldsymbol{H}^{\mathrm{f}}
$$

where the incidence matrix $\boldsymbol{D}^{\mathrm{f}}$ presents the relation between the interface tractions and displacements of a CNT fiber in the local Cartesian coordinate system. In the repeated identical subdomain approach, it needs to be calculated only once for all identical fibers. Based on (3) and (5), all boundary node values of the unknown variables can be solved using FM-BEM.

\section{Effective Elastic Modulus of CNT Composites}

The CNT composites can be simplified as transversely isotropic material. The average direction of CNT fibers is taken as $x_{1}$, which is also the direction of longer side of the RVE, and the RVE is stretched along this direction under uniform traction. The equivalent homogenized strains and stresses can be defined as:

$$
\begin{aligned}
& \bar{\varepsilon}_{i j}=\frac{1}{V} \int_{V} \varepsilon_{i j} \mathrm{~d} V=\frac{1}{2 V} \int_{S_{0}}\left(u_{i} n_{j}+u_{j} n_{i}\right) \mathrm{d} S \\
& \bar{\sigma}_{i j}=\frac{1}{V} \int_{V} \sigma_{i j} \mathrm{~d} V=\frac{1}{V} \int_{S_{0}} \sigma_{i k} x_{j} n_{k} \mathrm{~d} S
\end{aligned}
$$

where $V$ and $S_{0}$ are the volume and the surface of the RVE respectively. For the case of computation, it is taken that $\bar{\sigma}_{22}=\bar{\sigma}_{33}=0$; therefore the most interested Young's modulus can be identified as

$$
\bar{E}_{11}=\bar{\sigma}_{11} / \bar{\varepsilon}_{11}
$$

\section{Numerical Examples with Ideal Interfacial Conditions}

\section{1) Effect of Aspect Ratio of CNT Fibers on Effective Young's Modulus of CNT Composites}

The diameter of the CNT fiber is fixed as $D=1.4 \mathrm{~nm}$, and the volume of the CNT is considered to be $37 \%$ of the effective fiber's volume. The aspect ratio $L / D$ of the effective elastic fiber is varied from 10 to 90 . For the matrix material, the NASA LaRC-SI polymer (mentioned in Refs. [4, 5]) is used, with Young's modulus $E_{\mathrm{m}}=3.8 \mathrm{GPa}$ and Poisson's ratio $v_{\mathrm{m}}=$ 0.4. All the above parameters are adopted from Ref. [5] for the convenience of comparison. The elastic properties of effective elastic CNT fiber are taken as $v_{\mathrm{f}}=0.4$ and $E_{\mathrm{f}}=450.4 \mathrm{GPa}$, which is 100 times larger than $E_{\mathrm{m}}$. The RVE is taken as a rectangular parallelepiped with the side ratio as $L_{m x}: L_{m y}: L_{m z}=$ 4:1:1. Its length scale $L_{m y}$ can be determined as

$$
L_{m y}=\left(\frac{n V_{\mathrm{CNT}}}{4 \phi}\right)^{1 / 3}
$$

where $n$ stands for the number of CNTs, $\phi$ the volume fraction, and $V_{\mathrm{CNT}}$ the real volume of one CNT.

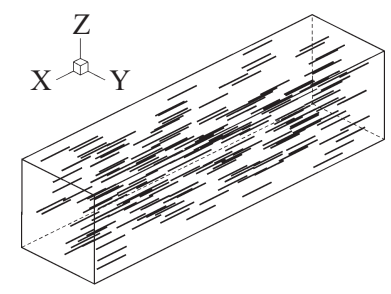

(a) aligned CNTs $(\mathrm{L} / \mathrm{D}=20)$

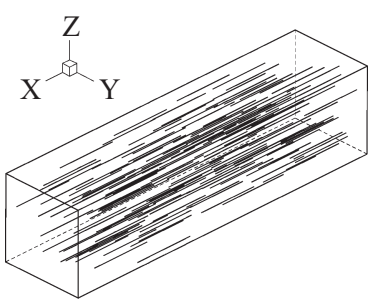

(c) aligned CNTs $(\mathrm{L} / \mathrm{D}=50)$

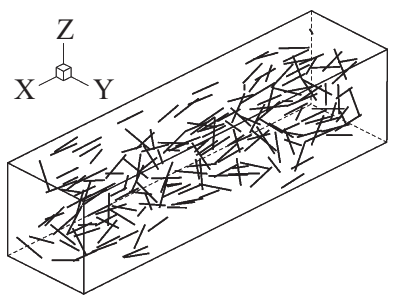

(b) randomly oriented $(\mathrm{L} / \mathrm{D}=20)$

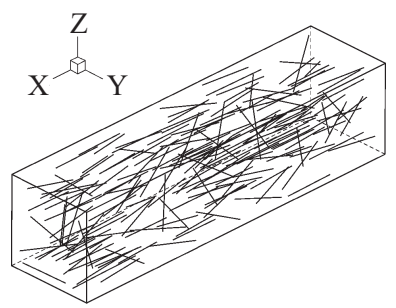

(d) randomly oriented $(\mathrm{L} / \mathrm{D}=50)$
Fig. 3. Some typical RVEs with 200 CNT fibers.

In the RVE, the effective elastic fibers are randomly distributed and separated, neither connection/intersection with others nor with the surfaces of RVE. Some typical RVEs are shown in Fig. 3.

The boundary conditions prescribed on six surfaces of the RVE are corresponding to the state of uniaxial tension,

$$
\begin{array}{ll}
t_{\mathrm{n}}=P, t_{\mathrm{s}}=t_{\mathrm{t}}=0 & \text { for } x=L_{\mathrm{m} x} \\
t_{\mathrm{n}}=0, t_{\mathrm{s}}=t_{\mathrm{t}}=0 & \text { for } y=L_{\mathrm{m} y} \\
t_{\mathrm{n}}=0, t_{\mathrm{s}}=t_{\mathrm{t}}=0 & \text { for } z=L_{\mathrm{m} z} \\
u_{\mathrm{n}}=0, t_{\mathrm{s}}=t_{\mathrm{t}}=0 & \text { for } x=0 \\
u_{\mathrm{n}}=0, t_{\mathrm{s}}=t_{\mathrm{t}}=0 & \text { for } y=0 \\
u_{\mathrm{n}}=0, t_{\mathrm{s}}=t_{\mathrm{t}}=0 & \text { for } z=0
\end{array}
$$

In such cases, the effective Young's modulus can be calculated simply as

$$
E_{\mathrm{e}}=\frac{P L_{\mathrm{m} x}}{\bar{u}}
$$

where $\bar{u}$ stands for the average normal displacement of the loaded surface $x=L_{\mathrm{m} x}$.

The results of the ratio $E_{\mathrm{e}} / E_{\mathrm{m}}$ versus the aspect ratio $L / D$ for the case of volume fraction $\phi=3.7 \%$ are shown in Fig. 4. It can be observed that the effective modulus increases with the increase of aspect ratio $L / D$ and approaches a limited value. Obviously, for the same aspect ratio and volume fraction the effective modulus for CNT composites with aligned CNTs are higher than that with randomly oriented CNTs.

\section{2) Effect of the Number of CNTs in a RVE}

For a group of test samples of RVE with prescribed aspect 


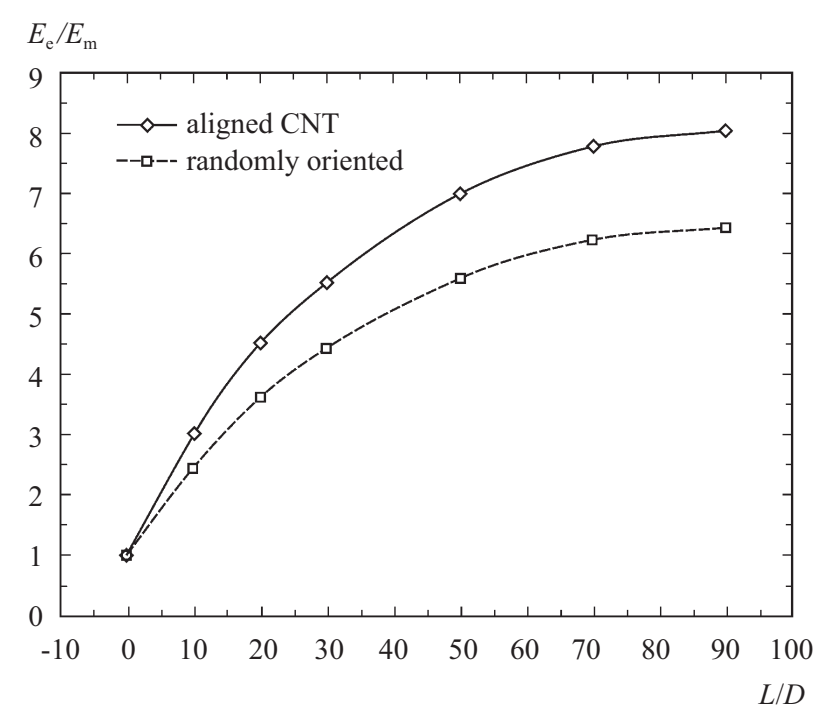

Fig. 4. Numerical results of the modulus ratio $E_{\mathrm{e}} / E_{\mathrm{m}}$ versus the aspect ratio $L / D$.

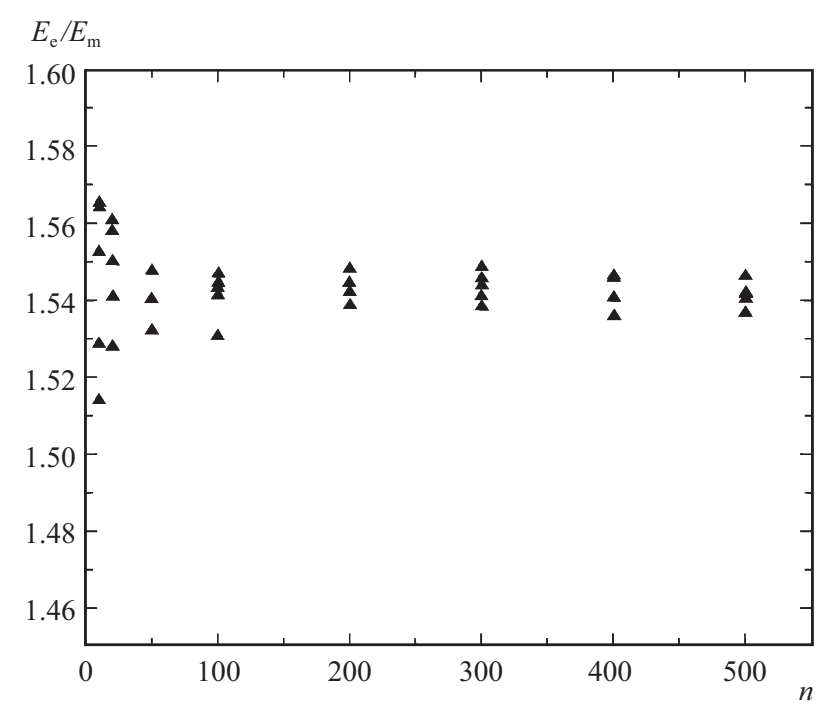

Fig. 5. Numerical results of the ratio of modulus $E_{\mathrm{e}} / E_{\mathrm{m}}$ versus the number of CNTs $n$ in RVE.

ratio, volume fraction, and number of CNTs, and with the same material properties of matrix and CNTs, the effective elastic modulus obtained could still be diverse. To ensure the reliability of the simulation results, a RVE should contain sufficient number of CNTs. As a test, several groups of RVE with prescribed aspect ratio $L / D=10$, volume fraction $\phi=5 \%$, and the material properties $E_{\mathrm{m}}=3.8 \mathrm{GPa}, E_{\mathrm{f}} / E_{\mathrm{m}}=50$ and $v_{\mathrm{f}}=$ $v_{\mathrm{m}}=0.4$, have been simulated.

The numerical results of the modulus ratio $E_{\mathrm{e}} / E_{\mathrm{m}}$ versus the number of CNT fibers in RVE are shown in Fig. 5. It can be observed that as the number of CNT fibers in a RVE reaches 200 the divergence of the modulus ratio is approximately equal to $1 \%$, which is acceptable for practical application.

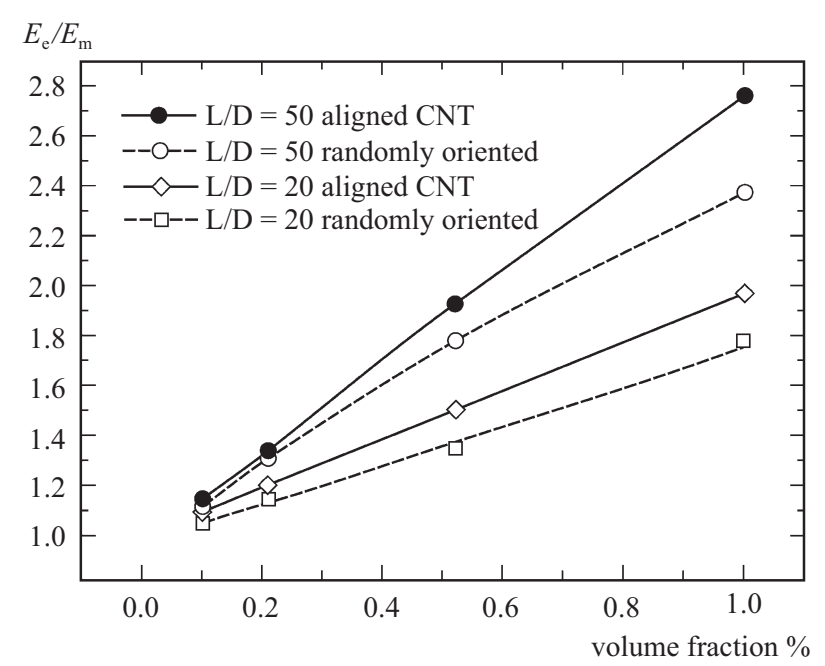

Fig. 6. Numerical results of the ratio of modulus $E_{\mathrm{e}} / E_{\mathrm{m}}$ versus the volume fraction of CNT.

Therefore in further investigation below, it is always taken 200 CNTs in a RVE.

At the same time, it can be seen that for simulating the mechanical property of practical CNT composites, the RVE contains only few CNT fiber is not sufficient, which cannot describe the effect of the distribution of CNTs correctly.

3) Effect of the Volume Fraction of CNTs on the Effective Young's Modulus of CNT Composites

For the design of CNT composites, the effect of the volume fraction of CNTs on the effective Young's modulus is most interested. In the simulation, both RVE with aligned and randomly oriented CNTs have been considered. The parameters of the CNTs are $D=1.4 \mathrm{~nm}, E_{\mathrm{f}}=450.4 \mathrm{GPa}, v_{\mathrm{f}}=0.4$, and for the matrix, $E_{\mathrm{m}}=3.8 \mathrm{GPa}, v_{\mathrm{m}}=0.4$. The aspect ratio $L / D$ are taken as 50 and 20 respectively.

The results of the effective Young's modulus versus the volume fraction of CNT are shown in Fig. 6. It is evident that the CNT composites with aligned CNTs have higher effective Young's modulus than that with randomly oriented CNTs, and the larger aspect ratio of CNTs gives higher effective modulus.

\section{4) Comparison with Other Numerical Results}

For the convenience of comparison, SWNT/LaRC-SI composite is simulated, and all parameters are adopted from Ref. [5]. The length of the CNT fibers is fixed at $50 \mathrm{~nm}$, and the radius of the CNT is $0.7 \mathrm{~nm}$. For the matrix material, the NASA LaRC-SI polymer is used, with Young's modulus of 3.8 $\mathrm{GPa}$ and Poisson's ratio of 0.4. The Poisson's ratio of CNT is taken as 0.4 , and the Young's modulus of CNT is $380 \mathrm{GPa}$, which is 100 times of the matrix modulus.

The effect of the CNT volume fraction on the effective elastic modulus of the CNT composites for aligned oriented case has been investigated. The results are plotted in Fig. 7, and compared with other results in Refs. $[4,5]$. Wherein the 


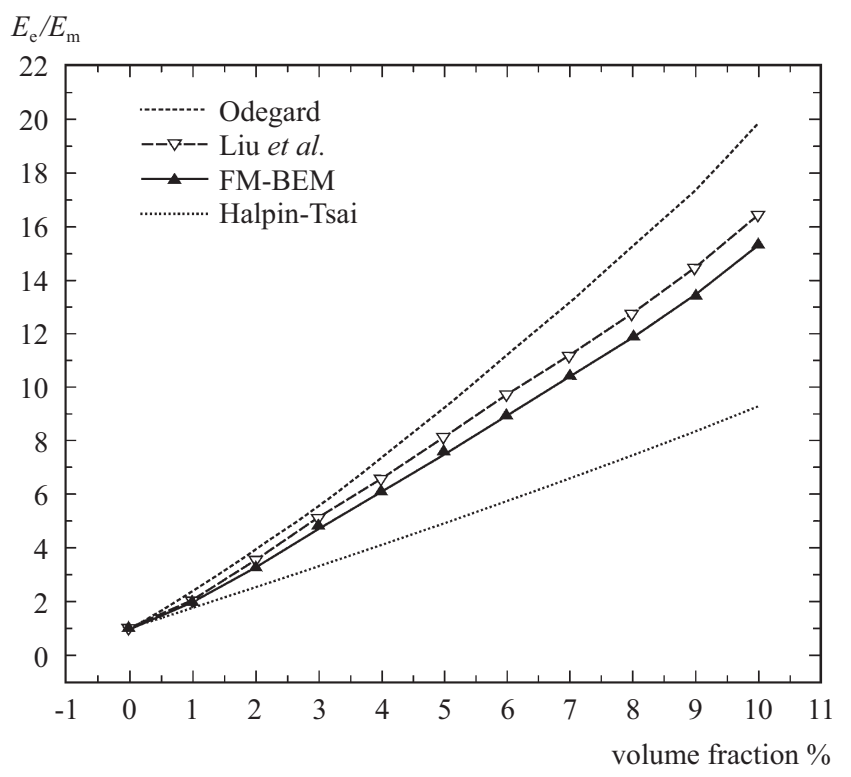

Fig. 7. Numerical results by presented FM-BEM in comparison with other results in literature.

results by Liu et al. are obtained also by the numerical simulation using fast multipole boundary element method, but based on the rigid-inclusion model rather than the effective elastic fiber model. The satisfied agreement with the results of Liu et al. could be observed. Odegard's results are based on the MD and an equivalent-continuum model, and the curve by Halpin-Tsai equation of a simplified model is also shown in the figure for reference.

The difference between the results obtained and those by Liu et al. [4] may be caused by the following factors: First, in Liu's work, CNT is considered as rigid-inclusion, but elastic in our model; second, the RVE in Liu's work is embedded in the infinite domain of matrix material, and what they solved is an exterior problem. In this work the model with symmetrical boundary conditions is applied, and this is more realistic but has to spend more computational resources. In addition the difference of loading mode will also bring slight difference to the results. Finally, the largest model of Liu's contains 16000 CNT rigid fibers, and the computation run on a super computer, but the above mentioned model contains only 200 CNT fibers and run on a cheaper PC cluster.

\section{5) Comparison with Experimental Results in Literature}

For comparison with the experimental results presented by Odegard, SWNT/LaRC-CP2 composites are simulated, and most of the parameters are adopted from Ref. [5]. For the matrix material, the NASA LaRC-CP2 polymer is used, with Young's modulus of $0.85 \mathrm{GPa}$ and Poisson's ratio of 0.4 . The Poisson's ratio of CNT is taken as 0.4, and the Young's modulus of CNT is $450.4 \mathrm{GPa}$, which is greater than 100 times of the matrix modulus. The length of the CNT fibers is fixed at $200 \mathrm{~nm}$, and the diameter of the CNT is taken as $2.8 \mathrm{~nm}$.

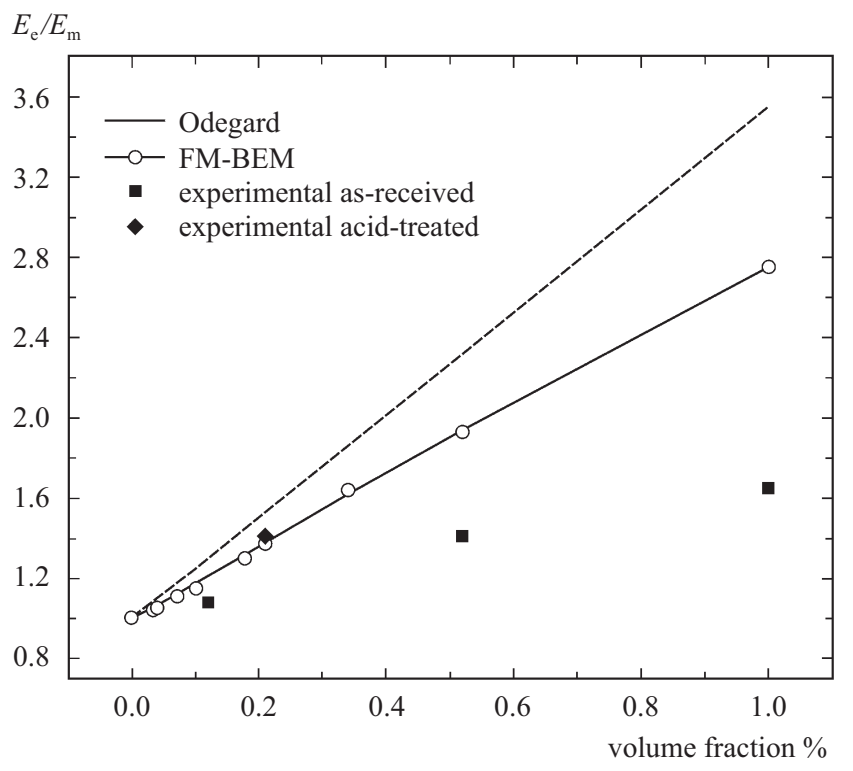

Fig. 8. Young's modulus of CNT reinforced LaRC-CP2 composite vs. CNT volume fraction.

Although good agreement between the numerical results and the experimental data for acid-treated CNT composites has been observed in Fig. 8, the predicted values of modulus are larger than the measured values for the as-received CNT, especially for values of CNT volume fraction greater than about $0.5 \%$. The difference between the experiments and the simulation is most likely caused by the following factors: First, the ideal interfacial condition is considered in the simulation model, the stiffness may be decreased by the transfer layer between CNT and matrix. Second, the model assumes that the effective fibers are perfectly dispersed in the polymer matrix, but a significant amount of CNTs may remain in bundles in the composite material. In addition, the CNT is considered as isotropic elastic fiber, but CNT is more close to being transversely iso-tropic material. Furthermore, the long CNTs will not all remain straight in the matrix. The closer agreement between the simulation and experimental data for the case with acid-treated CNTs indicates that the CNT are more dispersed in the case of acid-treated than in the case of asreceived.

\section{Two Models with Nonideal Interfacial Conditions}

As mentioned above, the difference between the experiments and the numerical results of effective Young's modulus is most likely caused by the reason that the interfacial condition between the CNT and matrix is not as strong as the ideal interfacial condition considered in the model, the stiffness may be decreased by the transfer layer between CNT and matrix. To improve the simulated elastic properties, two revised interfacial models have been proposed: one is elastic interface model (Fig. 9(a)), the another is embedded interfacial layer model (Fig. 9(b)). 


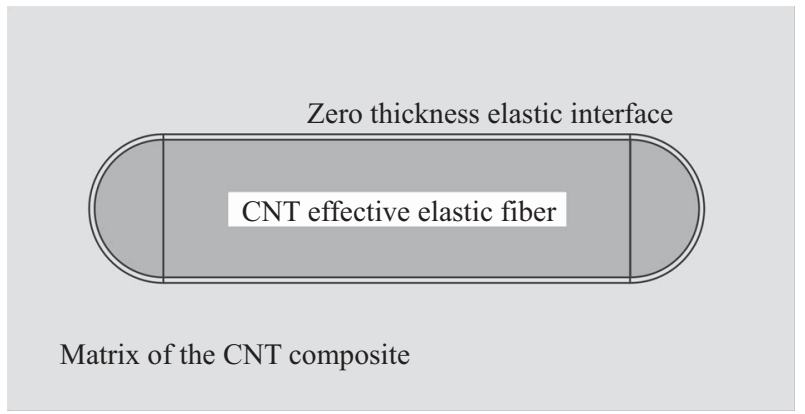

(a) elastic interfacial model

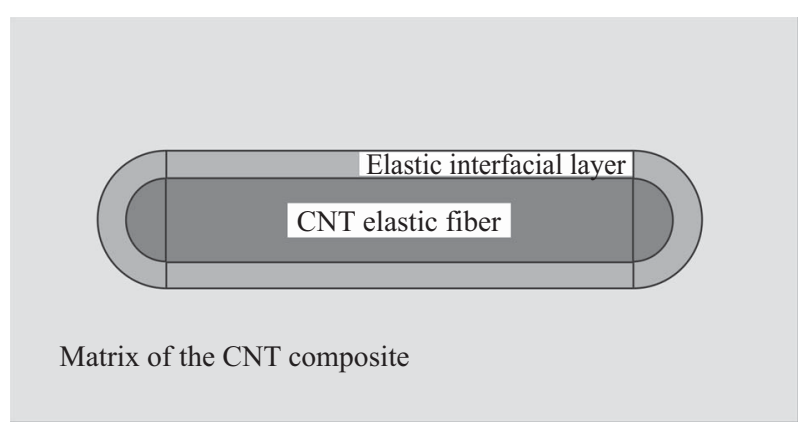

(b) embedded interfacial layer

Fig. 9. Revised interfacial models for simulating elastic property.

For the elastic interfacial model, the elastic interfacial condition can be written as

$$
\boldsymbol{T}_{k}^{0 \mathrm{e}}=-\boldsymbol{T}_{k}^{\mathrm{fe}}=\boldsymbol{K}^{\mathrm{e}}\left(\boldsymbol{U}_{k}^{0 \mathrm{e}}-\boldsymbol{U}_{k}^{\mathrm{fe}}\right)
$$

where the superscripts 0 and $\mathrm{f}$ denote the matrix side and the CNT fiber side for the interfacial variables, the superscript e denotes "for an element", and $\boldsymbol{K}^{\mathrm{e}}$ is the element interfacial elastic matrix. Therefore, the incidence matrix $\boldsymbol{D}^{\mathrm{f}}$ in (5) should be modified as

$$
\boldsymbol{D}^{\mathrm{f}}=\left[\boldsymbol{I}-\boldsymbol{K}^{-1}\left(\boldsymbol{G}^{\mathrm{f}}\right)^{-1} \boldsymbol{H}^{\mathrm{f}}\right]^{-1}\left[\left(\boldsymbol{G}^{\mathrm{f}}\right)^{-1} \boldsymbol{H}^{\mathrm{f}}\right]
$$

where $\boldsymbol{K}$ stands for the interfacial elastic matrix and can be integrated by element interfacial elastic matrix,

$$
\boldsymbol{K}=\left[\begin{array}{cccc}
\boldsymbol{K}^{\mathrm{e}} & 0 & \cdots & 0 \\
0 & \boldsymbol{K}^{\mathrm{e}} & \cdots & 0 \\
\vdots & \vdots & \ddots & \vdots \\
0 & 0 & \cdots & \boldsymbol{K}^{\mathrm{e}}
\end{array}\right] \quad \boldsymbol{K}^{\mathrm{e}}=\left[\begin{array}{ccc}
k_{1} & 0 & 0 \\
0 & k_{2} & 0 \\
0 & 0 & k_{3}
\end{array}\right]
$$

For simplicity, it is assumed that $k_{1}=k_{2}=k_{3}=k$.

For the embedded interfacial layer model, the governing equations derived from the boundary integral equations of matrix, CNT fiber and interfacial layer are combined by ideal interfacial conditions. The equations for the $k$-th CNT fiber and the corresponding interfacial layer can be written as

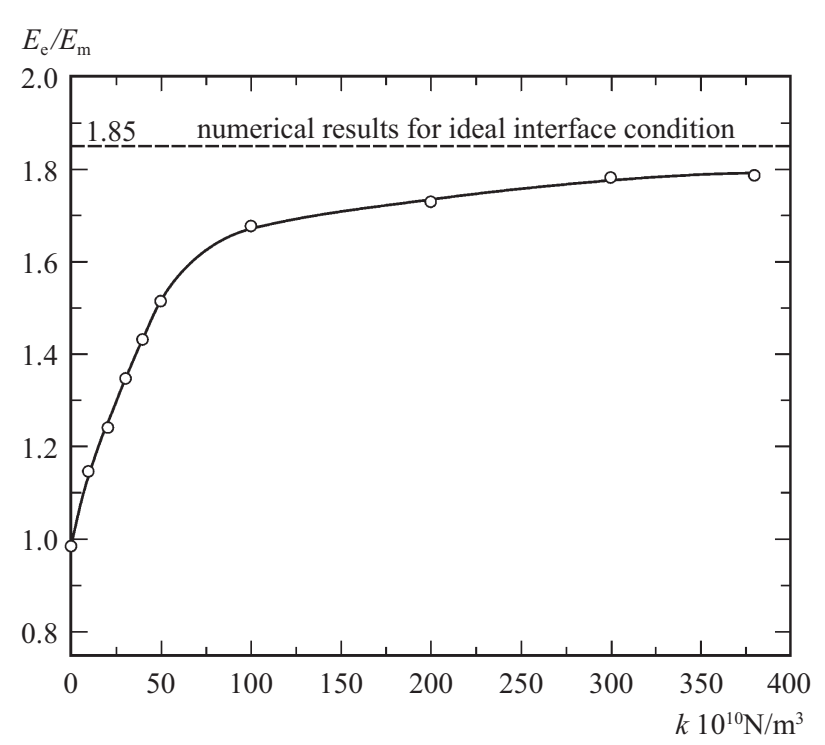

Fig. 10. The effect of interfacial elastic coefficient on the effective Young's modulus.

$$
\begin{aligned}
& \boldsymbol{H}^{\mathrm{f}} \boldsymbol{U}_{k}^{\mathrm{f}}=\boldsymbol{G}^{\mathrm{f}} \boldsymbol{T}_{k}^{\mathrm{f}} \\
& {\left[\begin{array}{ll}
\boldsymbol{H}_{11}^{\mathrm{i}} & \boldsymbol{H}_{12}^{\mathrm{i}} \\
\boldsymbol{H}_{21}^{\mathrm{i}} & \boldsymbol{H}_{22}^{\mathrm{i}}
\end{array}\right]\left\{\begin{array}{l}
\boldsymbol{U}_{k}^{0} \\
\boldsymbol{U}_{k}^{\mathrm{f}}
\end{array}\right\}=\left[\begin{array}{ll}
\boldsymbol{G}_{11}^{\mathrm{i}} & \boldsymbol{G}_{12}^{\mathrm{i}} \\
\boldsymbol{G}_{21}^{\mathrm{i}} & \boldsymbol{G}_{22}^{\mathrm{i}}
\end{array}\right]\left\{\begin{array}{c}
-\boldsymbol{T}_{k}^{0} \\
-\boldsymbol{T}_{k}^{\mathrm{f}}
\end{array}\right\}}
\end{aligned}
$$

where the superscripts 0 and f related to $\boldsymbol{U}$ and $\boldsymbol{T}$ denote the matrix side and the CNT fiber side for the interfacial variables, and the superscripts f and i related to $\boldsymbol{H}$ and $\boldsymbol{G}$ denote the matrices related to the fiber and interfacial layer. Therefore, the incidence matrix $\boldsymbol{D}^{\mathrm{f}}$ in (5) should be modified as

$$
\begin{aligned}
\boldsymbol{D}^{\mathrm{f}}= & {\left[\boldsymbol{G}_{21}^{\mathrm{i}}-\left(\boldsymbol{G}_{22}^{\mathrm{i}}\left(\boldsymbol{G}^{\mathrm{f}}\right)^{-1} \boldsymbol{H}^{\mathrm{f}}+\boldsymbol{H}_{22}^{\mathrm{i}}\right)\left(\boldsymbol{G}_{12}^{\mathrm{i}}\left(\boldsymbol{G}^{\mathrm{f}}\right)^{-1} \boldsymbol{H}^{\mathrm{f}}+\boldsymbol{H}_{12}^{\mathrm{i}}\right)^{-1} \boldsymbol{G}_{11}^{\mathrm{i}}\right]^{-1} } \\
& {\left[\boldsymbol{H}_{21}^{\mathrm{i}}-\left(\boldsymbol{G}_{22}^{\mathrm{i}}\left(\boldsymbol{G}^{\mathrm{f}}\right)^{-1} \boldsymbol{H}^{\mathrm{f}}+\boldsymbol{H}_{22}^{\mathrm{i}}\right)\left(\boldsymbol{G}_{12}^{\mathrm{i}}\left(\boldsymbol{G}^{\mathrm{f}}\right)^{-1} \boldsymbol{H}^{\mathrm{f}}+\boldsymbol{H}_{12}^{\mathrm{i}}\right)^{-1} \boldsymbol{H}_{11}^{\mathrm{i}}\right] }
\end{aligned}
$$

By using the repeated identical subdomain approach, it needs to be calculated only once for all identical fibers with interfacial layer. In this way the efficiency can be enhanced remarkably.

\section{Numerical Examples with Nonideal Interfacial Conditions}

\section{1) Effect of Interfacial Elastic Coefficient $k$ on Effective Young's Modulus of CNT Composites}

In the simulation, the RVE with 200 aligned CNTs with interfacial elastic conditions has been considered. The parameters of the CNTs are $D=1.4 \mathrm{~nm}, E_{\mathrm{f}}=450.4 \mathrm{GPa}, \nu_{\mathrm{f}}=0.4$, and for the matrix, $E_{\mathrm{m}}=3.8 \mathrm{GPa}, v_{\mathrm{m}}=0.4$. The aspect ratio $L / D$ is taken as 20, and the volume fraction of the CNT is $1 \%$.

The obtained results of ratio of Young's modulus $E^{\mathrm{e}} / E^{\mathrm{m}}$ versus the interfacial elastic coefficient $k$ are shown in Fig. 10. 
It can be observed that the effective Young's modulus increases with the increase of interfacial elastic coefficient, and approaches the value for the case with ideal interface condition.

\section{2) Comparison with Experimental Results in Literature}

In order to reduce the difference between the numerical results obtained and the experimental data for CNT composite with as-received CNTs in Ref. [5], the RVEs with 200 aligned CNTs with both interfacial elastic conditions and embedded interfacial elastic layer have been investigated. The parameters of the CNTs are $L=200 \mathrm{~nm}, E_{\mathrm{f}}=450.4 \mathrm{GPa}, v_{\mathrm{f}}=0.4$, and for the matrix, $E_{\mathrm{m}}=0.85 \mathrm{GPa}, v_{\mathrm{m}}=0.4$. The volume fraction of the CNT is $1 \%$. For the elastic interface model, the diameter of the effective CNT fiber is $D=2.8 \mathrm{~nm}$, and the interfacial elastic coefficient $k=5 \times 10^{11} \mathrm{~N} / \mathrm{m}^{3}$. For the model of elastic interfacial layer, the diameter of the CNT fiber is 1.68 $\mathrm{nm}$, the outer diameter of the interfacial layer is $2.8 \mathrm{~nm}$, and the material properties of the layer are $E_{\mathrm{i}}=50 \mathrm{GPa}, v_{\mathrm{i}}=0.4$.

The numerical results of the Young's modulus ratio $E_{\mathrm{e}} / E_{\mathrm{m}}$ obtained are plotted in Fig. 11, and compared with other numerical and experimental results. It can be observed that the results for both models with nonideal interfacial conditions are much closer to the experimental data than that for model with ideal interfacial conditions, for the case with as-received CNTs.

All these computations are run on a PC cluster THPCC in Tsinghua National Laboratory for Information Science and Technology, with $1.3 \mathrm{GHz}$ and $4 \mathrm{~GB}$ memories per CPU. The example of largest scale has a total DOF of $3,159,148$, costs about $7.6 \mathrm{~h}$ using $12 \mathrm{CPUs}$.

\section{SIMULATION OF THE THERMAL AND ELECTRIC PROPERTIES}

For the simulation of thermo and electric properties, the CNTs are modeled as straight fibers with high thermal conductivity and high electric conductivity, respectively. It is simulated using large-scale parallel computation of fast multipole boundary element method, wherein the repeated subdomain approach is applied. Numerical examples are given to show the feasibility of the presented approach, and the comparison of the numerical and experimental results has shown remarkable difference. To reduce such difference more practical models considering different influencing factors are required.

\section{BEM for Simulating Thermal and Electric Properties of CNT Composites}

Instead of (1) and (2), the boundary integral equations for steady heat and electric conduction are expressed for the matrix and the $k$-th CNT fiber, respectively,

$$
\begin{gathered}
C(x) t(x)+\int_{S_{0}+\sum S_{k}} F^{0}(x, y) t(y) d S(y) \\
=\int_{S_{0}+\sum S_{k}} T^{0}(x, y) f(y) d S(y)
\end{gathered}
$$

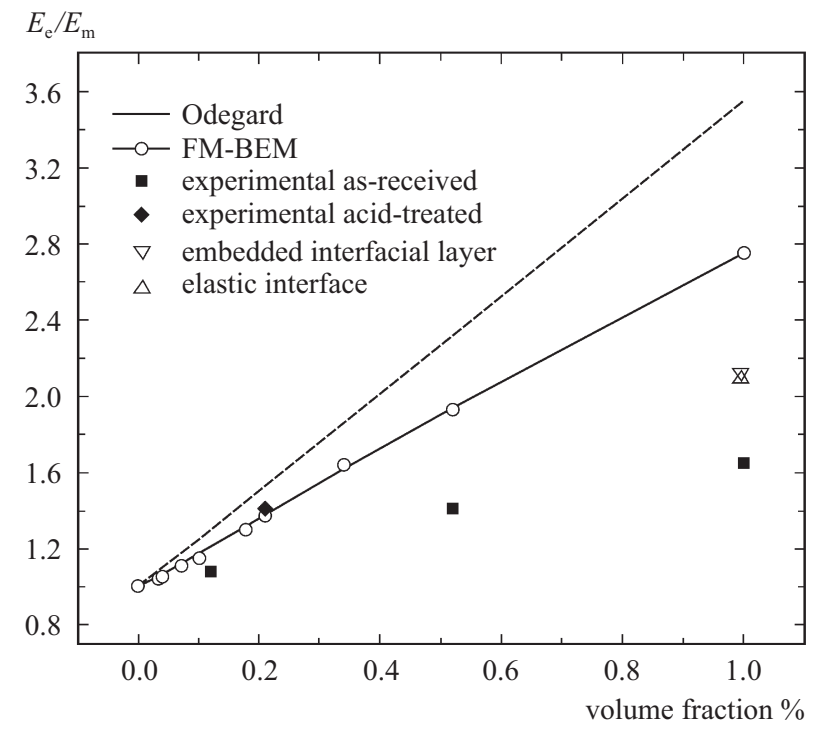

Fig. 11. The comparison of numerical results with experimental data.

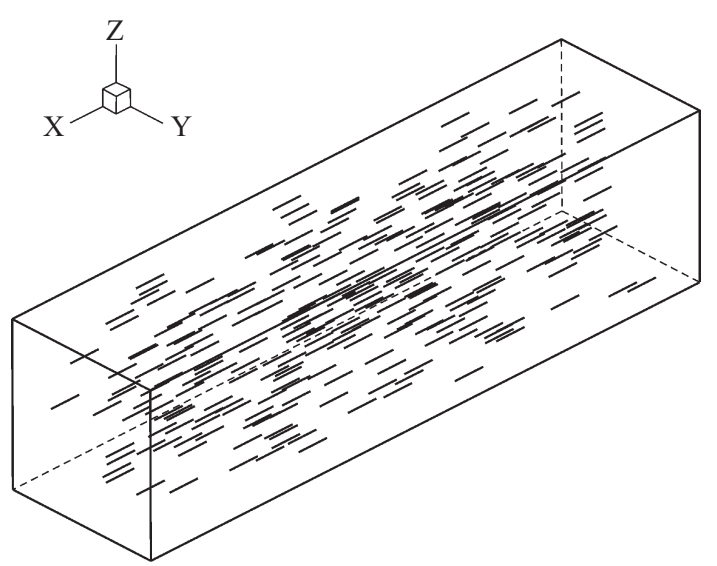

Fig. 12. A RVE containing 300 aligned CNTs.

$$
\begin{gathered}
C(x) t(x)+\int_{S_{k}} F^{k}(x, y) t(y) d S(y) \\
=\int_{S_{k}} T^{k}(x, y) f(y) d S(y)
\end{gathered}
$$

where $t$ denotes the boundary temperature or electric potential, $f$ denotes the normal heat flux or normal electric current, respectively for heat or electric conduction problem, $T(x, y)$ and $F(x, y)$ are the fundamental solutions of potential problem.

Based on the FM-BEM for simulating elastic property of $\mathrm{CNT}$ composites, the simulation of their thermal and electric properties can be performed correspondingly.

\section{Simulation of Thermal Properties}

Considering the limitation of computational resource on one PC, a RVE containing up to 300 CNTs is adopted. One global model of examples is shown in Fig. 12, with fiber aspect ratio and volume fraction equal to 20.0 and $0.15 \%$, respectively. 
thermal conductivity ratio

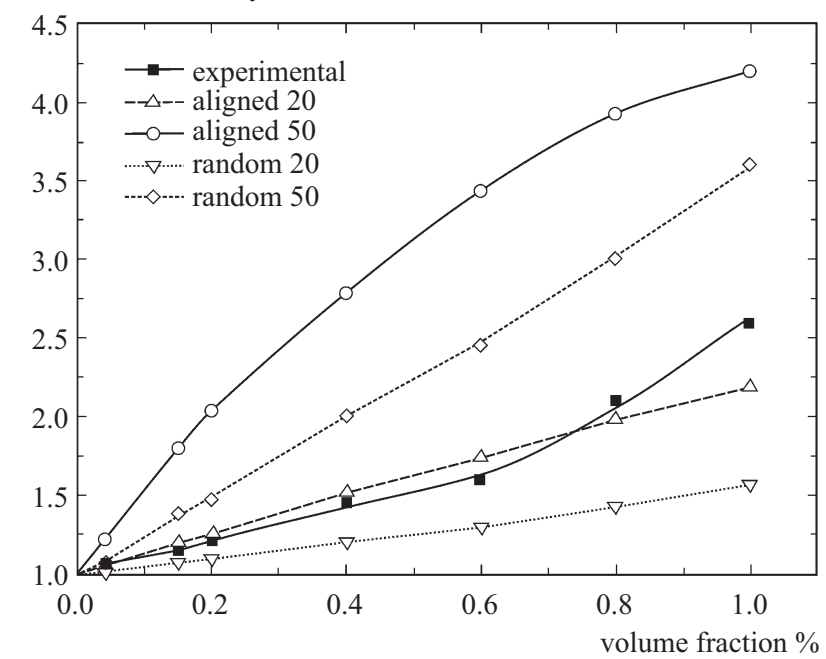

Fig. 13. Overall result view of thermal conductivities estimation in comparison with experimental data.

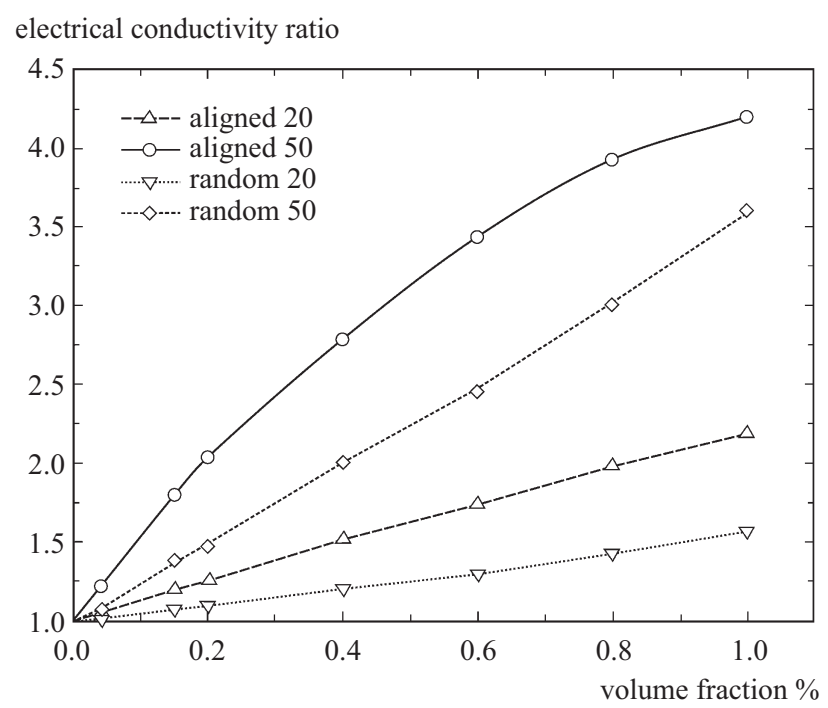

Fig. 14. Predicted effective conductivity of CNT-reinforced Composites.

There are 640 triangular constant elements for one CNT in the mesh, which yields 640 DOF per CNT fiber. This mesh was found to be sufficient for obtaining converged results for the estimated effective conductivity.

Figure 13 is an overall plot of four cases studied in this paper: the directions of CNTs are aligned or randomly oriented, the aspect ratios of CNTs equals 50 or 20. In all these cases, the effective thermal conductivity increases significantly when the volume fraction increases, with the fastest increase in the case that CNTs are aligned oriented and the aspect ratio is taken as 50. The plot shows that an enlargement of aspect ratio can obtain a significant thermal conductivity enhancement and aligned orientation of CNTs is also propitious for thermal conductivity enhancements.

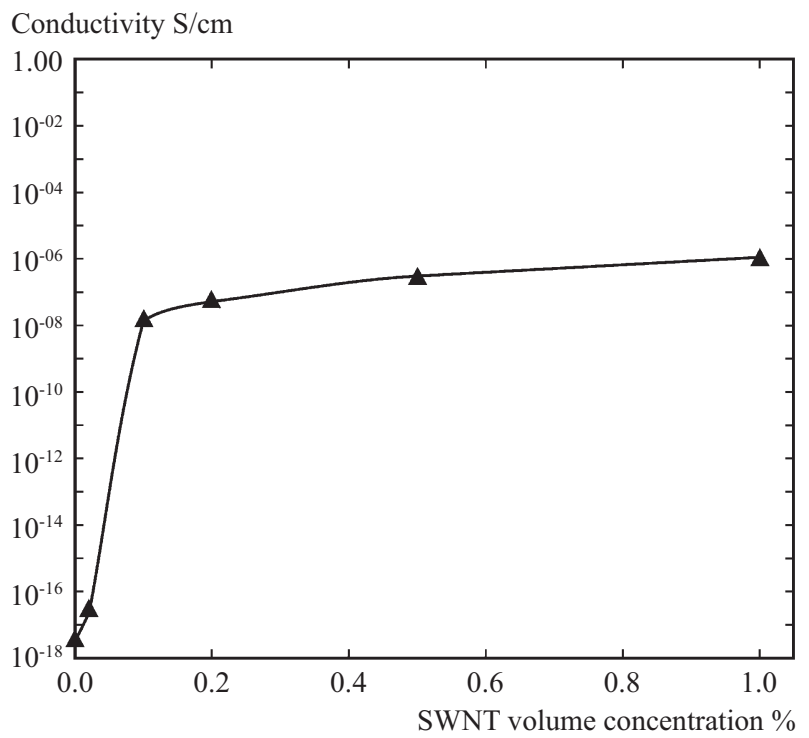

(a) in Ref. [6]

Conductivity $\mathrm{S} / \mathrm{cm}$

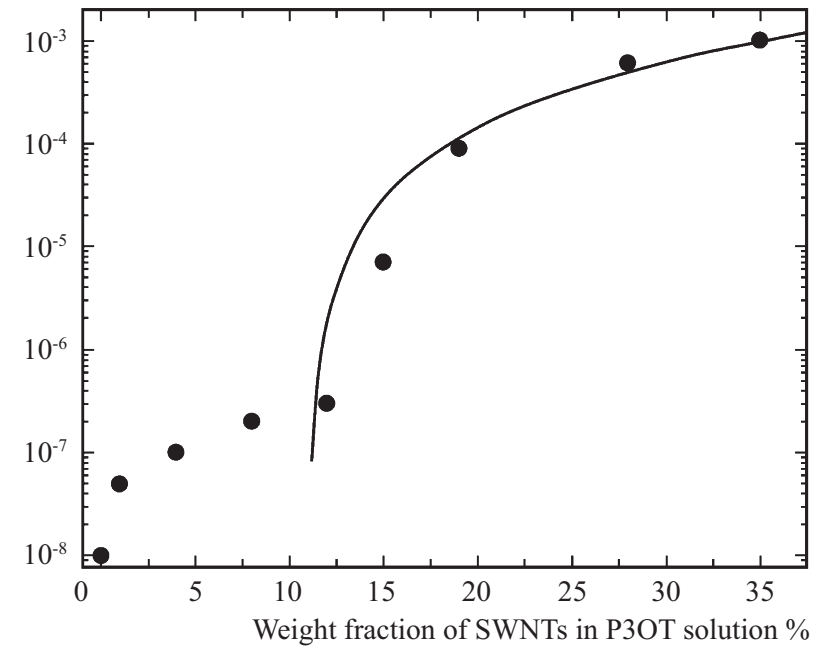

(b) in Ref. [2]

Fig. 15. Experimental data published in references.

The difference between the experiments data [1] and the numerical results obtained is most likely caused by the following factors: On the one hand, the thermal contact between the CNTs has not been considered in the computation, which will increase the thermal conductivity; on the other hand, in practical case the thermal contact condition of CNT-matrix interfaces should be imperfect rather than ideal condition considered in the numerical model adopted in this investigation, which will decrease the conductivity.

\section{Simulation of Electric Properties}

For the numerical estimation of electric property, it is shown from Fig. 14 that, there is a maximum of approximately $400 \%$ increase in the effective electric conductivity of aligned CNT composites with CNT aspect ratio of 50.0 in the range of CNT 
volume fraction up to $1.0 \%$. Increase of $\mathrm{CNT}$ aspect ratio results in an increase of effective electric conductivity. In addition, randomly oriented CNTs result in a decrease of effective electric conductivity.

While for experimental results [2, 6] shown in Fig. 15, it is observed that significant changes occur in a small scope of CNT concentration. The effective electric conductivity reaches to a value several orders higher than that of matrix with a very small CNT volume/weight fraction. The difference between the experiments and the simulation is mostly caused by the following factors: while the model assumes that CNTs are well separated from each other and will not cross the surface of matrix sub-domain, in practice a significant amount of CNTs remain in bundles in the matrix, contact each other or cross the boundary of matrix sub-domain, which greatly enhance the effective electric conductivity. The phenomena observed in real CNT composites should be reflected in further developed models.

\section{CONCLUDING REMARKS}

The numerical tests clearly demonstrate the potential of the FM BEM for large-scale computation of CNT composites. Using this method, the effective elastic, thermal and electric properties can be simulated, but the difference between the numerical and experimental results have been observed. That means the numerical models have to be improved to consider more practical micro-structural factors, including the imperfect CNT-matrix interface conditions and thermal and electric contact between CNTs. Two revised models with nonideal interfacial conditions are presented for the simulation of elastic properties, and verified. Based on the present work, future investigations will be carried out in further development of new models.

\section{ACKNOWLEDGMENTS}

Financial supports for the project from the National Natural Science Foundation of China, under grant No. 10472051, and for a project from Schlumberger Technologies (Beijing) Ltd., are gratefully acknowledged.

\section{REFERENCES}

1. Choi, S. U. S., Zhang, Z. G., Yu, W., Lockwood, F. E., and Grulke, E. A., "Anomalous thermal conductivity enhancement in nanotube suspensions," Applied Physics Letters, Vol. 79, pp. 2252-2254 (2001).

2. Kymakis, E., Alexandou, I., and Amaratunga, G. A. J., "Single-walled carbon nanotube-polymer composites: electrical, optical and structural investigation," Synthetic Metals, Vol. 127, pp. 59-62 (2002).

3. Lei, T., Yao, Z. H., Wang, H. T., and Wang, P. B., "A parallel fast multipole BEM and its applications to large- scale analysis of 3-D fiber- reinforced composites," Acta Mechanica Sinica, Vol. 22, pp. 225-232 (2006).

4. Liu, Y. J., Nishimura, N., Otani, Y., Takahashi, T., Chen, X. L., and Munakata, H., "A fast boundary element method for the analysis of fiber-reinforced composites based on a rigid-inclusion model," Journal of Applied Mechanics, Vol. 72, pp. 115-128 (2005).

5. Odegard, G. M., Gates, T. S., Wise, K. E., Park, C., and Siochi, E. J., "Constitutive modeling of nanotubes-reinforced polymer composites," Composite Science and Technology, Vol. 63, pp. 1671-1687 (2003).

6. Ounaies, Z., Park, C., Wise, K. E., Siochi, E. J., and Harrison, J. S., "Electrical properties of single wall carbon nanotube reinforced polyimide composites," Composites Science and Technology, Vol. 63, pp. 1637-1646 (2003).

7. Wang, H. T. and Yao, Z. H., "A new fast multipole boundary element method for large scale analysis of mechanical properties in 3D particlereinforced composites," Computer Modeling in Engineering \& Sciences, Vol. 7, pp. 85-96 (2005).

8. Wang, H. T. and Yao, Z. H., "Application of a new fast multipole BEM for simulation of 2D elastic solid with large number of inclusions," Acta Mechanica Sinica, Vol. 20, pp. 613-622 (2004). 